\title{
Analyzing a degenerate buffer with general inter-arrival and service times in discrete time
}

\author{
W. Rogiest $\cdot$ K. Laevens $\cdot$ J. Walraevens $\cdot$ H. Bruneel
}

Received: 25 September 2006 / Revised: 9 February 2007 / Published online: 7 June 2007

(C) Springer Science+Business Media, LLC 2007

\begin{abstract}
In novel switching approaches such as Optical Burst Switching, the involved buffers can only provide a degenerate waiting room, with delays restricted to multiples of a basic value, the granularity. Although the resulting performance loss was already studied analytically, previous work is either limited by the assumption of independent arrivals, or it involves a matrix with size growing fast with buffer size or arrival process complexity.

Overcoming this, we developed a generic and accurate loss performance model for a degenerate GI/G/1 buffer in discrete time, that yields results instantly for any constellation of burst sizes, inter-arrival times, granularity, load and buffer size. This paper presents our model and compares its results to simulations, illustrating the impact of different types of correlation in the arrival process on loss performance. Our basic model is general and accurate, it can thus serve as a basic tool for optical switch design.
\end{abstract}

Keywords Degenerate buffer - Optical buffer · FDL buffer - Cyclic waiting - Lakatos-type queueing system . Correlated traffic · Granularity · Discrete-time queueing . Generating functions

Mathematics Subject Classification (2000) $68 \mathrm{M} 20$

W. Rogiest $(\varangle) \cdot$ K. Laevens $\cdot$ J. Walraevens $\cdot$ H. Bruneel

SMACS Research Group, Ghent University,

Sint-Pietersnieuwstraat 41, 9000 Ghent, Belgium

e-mail: wouter.rogiest@ugent.be

K. Laevens

e-mail: k1@telin.ugent.be

J. Walraevens

e-mail: jw@telin.ugent.be

H. Bruneel

e-mail: hb@telin.ugent.be

\section{Introduction}

Within the tradition of queueing research, degenerate buffer systems were studied for the first time only recently. To the best of the authors' knowledge, Lakatos was the first to publish on the subject. He studied a degenerate $\mathrm{M} / \mathrm{M} / 1$ queue in continuous time in [1], and extended the analysis to a discrete-time setting in [2]. He based the model on a problem connected with the landing of airplanes, where arriving airplanes are obliged to wait for a discrete number of orbits of fixed length before landing. This system has a degenerate waiting room, which Lakatos refers to as cyclic waiting, while some more recent publications in this tradition [3] speak of a Lakatos-type queueing system. Important to note is that this system differs fundamentally from a retrial system [4], although some, like the one of [5], have a very similar setting. A general explanation of the differences can be found in [3].

A wide-spread interest in degenerate buffers was generated by the advent of optical networking. While optical fibers are the standard carrier for data transport over long distances, the data processing at the nodes is still mainly done in the electronic domain. To increase switching speeds in the long run, solutions like Optical Packet Switching (OPS) and Optical Burst Switching (OBS) aim to process data in the optical domain [6]. The involved optical buffers are implemented as a set of $N+1$ Fiber Delay Lines (FDLs), with lengths that are typically a multiple of a basic value $D$ called the granularity (a term coined in [7]). This results in a degenerate waiting room, with waiting times $0 \cdot D, 1 \cdot D, 2 \cdot D \cdots N \cdot D$.

Over the years, several authors have developed analytic models for such optical buffers, especially in the case of memoryless arrivals [7-11]. Since arrival processes in (optical) communication networks are known to be bursty, a sep- 
arate study of the impact of correlation in the arrival process on performance is crucial, but was only given attention to recently. Almeida et al. [12,13] characterize the system with $N+1$ possible waiting times as states of a Markov chain, and obtain exact results for memoryless inter-arrival times with the introduction of $N+1$ blocking states. However, for general inter-arrival times, they obtain approximate results, and need to introduce a multiple of $N+1$ blocking states (10 times $N+1$ in [13]) to obtain sufficient accuracy, which leads to a very large state space, complicating the calculation of waiting time and loss probabilities. Opposed to this, the results of the current contribution are analytic formulas, that have the benefit of being valid for a broad range of parameters at once, while yielding results instantly, even for large buffer sizes or exotically distributed inter-arrival times. Although valid for the same traffic assumptions as [13], the formulas are obtained by a completely different approach, based on the use of probability generating functions (pgf's). In this regard, it can be seen as an extension of [9], with the specific merit that the most restricting assumption in [9], that of a memoryless arrival process, is alleviated. As such, this contribution is the first to provide an exact analysis of the degenerate GI/G/1 queue of infinite size in discrete-time, while the continuous-time case still remains unstudied. The analysis is exact for an infinite-sized buffer, but it also offers accurate approximate results for finite-sized buffers, as validated in this paper by comparison with simulations. As such, this paper provides analytical formulas for the performance evaluation of optical buffers that apply to a wide variety of traffic settings.

In Sect. 2, we introduce the degenerate buffer setting and the modeling approach by means of pgf's. In Sect. 3, we foreground the crucial steps of the analysis, referring to Appendix for details at one point. The analytic performance measures, derived in Sect. 4, are compared with simulations in Sect. 5, where also the specific impact of correlation in the arrival process on loss performance is foregrounded.

\section{Stochastic model}

\subsection{Discrete-time setting}

The model we construct has a discrete-time setting, that assumes events take place synchronously, at the beginning of time slots, as is frequently proposed in the context of optical switching. This implies that all time-related variables and measures, including the granularity $D$, are expressed as multiples of the slot length, that may be arbitrary, and is therefore not mentioned explicitly in the remainder of the paper. In the unslotted case, inter-arrival times and

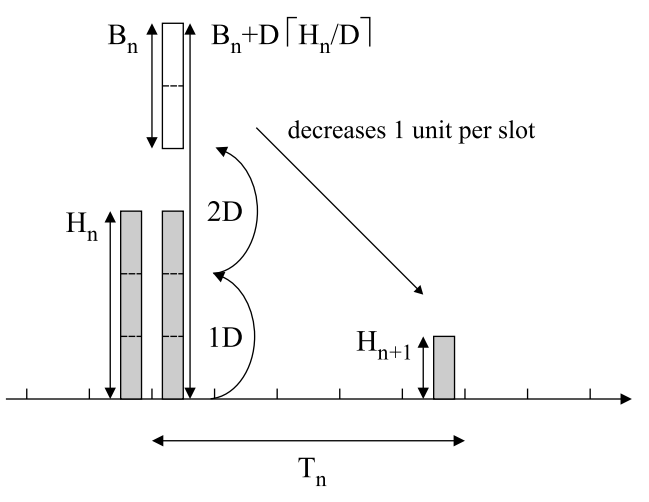

Fig. 1 Evolution of $H_{n}$ in time. A burst $B_{n}$ arrives, and has to wait for $D\left\lceil H_{n} / D\right\rceil$, so as to avoid contention with previously arrived bursts

service times may also be continuous (asynchronous). We note, however, that one can approximate the continuoustime case arbitrarily close if one takes a very small slot length, and scales the involved variables and measures accordingly.

\subsection{Degenerate buffer setting}

A degenerate buffer of size $N+1$ is a set of $N+1$ delay lines, with lengths expressed in a number of slots $0 \cdot D, 1$. $D \cdots N \cdot D$ and feeds into a single outgoing channel. Whenever a customer arrives in a non-empty buffer, it has to be queued at least until all previous customers have left the system (FIFO). Due to the degeneration of the buffer, customers have to wait longer, so as to wait for a time that is a multiple of $D$. This extra waiting time results in an under-utilization of the outgoing channel, reflected in the occurrence of socalled voids, i.e., periods during which the outgoing channel remains unused, despite the fact that the system is not empty. In case of a finite-sized buffer, the maximum waiting time is $N \times D$, and any customer requiring a larger delay is lost. As a consequence, finite-sized degenerate buffers suffer both under-utilization of the outgoing channel, as well as increased loss, if compared to a classical (non-degenerate) buffer. For most of the analysis, we will assume an infinitesized buffer $(N=\infty)$. Finite-sized buffers are considered in Sects. 4.2 and 5 .

\subsection{Scheduling horizon}

At this point, we focus on how arriving customers join the queue of a degenerate buffer. The relation between the involved random variables, all expressed in a number of slots, is illustrated in Fig. 1. Numbering customers in the order of their arrival, the inter-arrival time $T_{n}$ captures the time between the $n$th arrival instant and the next. The burst size $B_{n}$ is the service time of the $n$th customer. Upon arrival, customer $n$ has to wait for at least an amount $H_{n}$, the time 
needed for all previously arrived customers to be served. This amount we call the scheduling horizon $H_{n}$, as observed by the $n$th customer upon arrival. Due to the buffer's degeneration, the customer has to wait for a waiting time $W_{n}$, that is a multiple of $D$ and is sufficiently long, i.e., $W_{n} \geq H_{n}$.

The evolution of these variables can be captured in one system equation, namely

$H_{n+1}=\left\lceil D \cdot\left\lceil\frac{H_{n}}{D}\right\rceil+B_{n}-T_{n}\right]^{+}$.

This equation contains two non-linearities. Introducing some additional notation, we can split this equation into two parts. The first non-linearity is

$H_{n+1}=\left[G_{n}-T_{n}\right]^{+}$

with

$G_{n}=B_{n}+W_{n}$,

where $[x]^{+}=\max \{x, 0\}$, while the second one is

$W_{n}=D \cdot\left\lceil\frac{H_{n}}{D}\right\rceil$

In the following, we will refer to the non-linearity in (2) as the queueing effect, since it occurs frequently in classical queueing analysis. The non-linearity in (4) we will refer to as the granularity effect, since it reflects the system's degeneration.

\subsection{Traffic model}

To proceed with the analysis of (2) and (4), we impose certain restrictions on the burst sizes $B_{n}$ and inter-arrival times $T_{n}$. We assume both to form a sequence of independent and identically distributed (iid) random variables (rv's). In our analysis, we use the probability generating function (pgf) of the probability mass function (pmf) of the involved variables. The burst sizes $B_{n}$, e.g., have a common pmf $\operatorname{Pr}\left[B_{n}=k\right](k=1,2 \ldots)$ and a pgf

$$
B(z)=\mathrm{E}\left[z^{B_{n}}\right]=\sum_{k=1}^{+\infty} z^{k} \operatorname{Pr}\left[B_{n}=k\right]
$$

and likewise for the other variables $T_{n}, H_{n}, G_{n}$ and $W_{n}$.

\section{Analysis for the infinite system}

The analysis assumes a system of infinite size. The approach consists in solving queueing effect and granular- ity effect separately. A crucial third step is then to combine both correctly, which requires involving Rouché's theorem.

\subsection{Conditions}

From this point on, we assume that the system is stable. The condition for stability is investigated below in Sect. 4.1. Under this condition, the distributions of $H_{n}$ (and likewise, for the other variables $G_{n}$ and $W_{n}$ ) converge, for $n \rightarrow \infty$, to a unique stochastic equilibrium distribution, that no longer relates to the initial condition of the system. Associated with this distribution is a common rv $H$, and a pgf $H(z)$, of which we will derive the explicit form in the following. For the burst time distribution, we assume that the mean value of the burst size is finite, $\mathrm{E}[B]<\infty$, which is not a very restrictive assumption. For the inter-arrival time distribution, we assume that its pgf $T(z)$ is rational. Both the numerator and denominator are thus polynomials of finite degree. This assumption too is not very restrictive from a modeling point of view, and includes the distributions treated in Sect. 5.

\subsection{The queueing effect}

Since we assume the system is stable, we analyze the queueing effect as it occurs for the stochastic equilibrium distributions, and (2) becomes

$H=[G-T]^{+}$

We emphasize that $G$ and $T$ (and, $G_{n}$ and $T_{n}$ ) are statistically independent, which is essential to our current analysis. Now, the complexity of a transform-based solution to this problem depends critically on the exact form of the distribution, or equivalently, the pgf of $T$, as discussed in for example [14].

To solve (5), we write the rational pgf of $T$ as

$T(z)=\frac{N(z)}{P(z)}=\frac{\sum_{i=0}^{N} n_{i} z^{i}}{\sum_{i=0}^{P} p_{i} z^{i}}$.

In other words, we label the numerator as a polynomial $N(z)$ of degree $N$, and the denominator as a polynomial $P(z)$ of degree $P$. Under these assumptions, (6) lends itself to an exact analysis, as explained in Appendix. In appendix is explained how. The result in terms of pgf's reads

$H(z)=G(z) T\left(z^{-1}\right)+T^{*}(1)-T^{*}\left(z^{-1}\right)$.

Here, $T^{*}(z)$ is an auxiliary function that is rational, just like $T(z)$, but not a pgf, as $T^{*}(z) \neq 1$. It shares the denominator of $T(z), P(z)$, but differs in its numerator $N^{*}(z) \neq N(z)$, 
that is a polynomial of degree $N$ or less. In accordance with (6), we can thus write

$T^{*}(z)=\frac{N^{*}(z)}{P(z)}=\frac{\sum_{i=0}^{N} n_{i}^{*} z^{i}}{\sum_{i=0}^{P} p_{i} z^{i}}$.

We note that the coefficients $n_{i}^{*}$ are unknown. However, applying Rouché's theorem in the following will enable us to proceed without having to determine them explicitly. We focus now on the second non-linearity.

\subsection{The granularity effect}

The granularity effect reflects the degeneration of the buffer, that is only able to realize delays that are a multiple of the granularity $D$. It is captured by

$W=D \cdot\left\lceil\frac{H}{D}\right\rceil$.

In previous work [9] it was obtained that

$W(z)=\sum_{k=0}^{D-1} \frac{1}{D} \frac{z^{D}-1}{z \epsilon_{k}-1} H\left(z \epsilon_{k}\right)$,

where the symbols $\epsilon_{k}$ denote the $D$ different complex $D$ th roots of unity, i.e., $\epsilon_{k}=e^{j 2 \pi k / D}(k=0, \ldots, D-1)$. A property of this solution is that it is "periodic" in the $z$-plane, in that $W\left(z \epsilon_{k}\right)=W(z), k=0, \ldots, D-1$, which is a direct consequence of the fact that $W(z)$ is a function of $z^{D}$ only.

\subsection{Combining results}

We now start from (7), and apply the property that

$G(z)=B(z) W(z)$.

This is so, because of (3), and the fact that the pgf of the sum of two independent rv's is the product of their separate pgf's. We find that

$$
H(z)=B(z) W(z) T\left(z^{-1}\right)+T^{*}(1)-T^{*}\left(z^{-1}\right) .
$$

Further, substituting this expression for $H(z)$ into (10), we find a functional equation for $W(z)$,

$$
\begin{aligned}
W(z)= & \sum_{k=0}^{D-1} \frac{1}{D} \frac{z^{D}-1}{z \epsilon_{k}-1}\left\{B\left(z \epsilon_{k}\right) W\left(z \epsilon_{k}\right) T\left(\left(z \epsilon_{k}\right)^{-1}\right)\right. \\
& \left.+T^{*}(1)-T^{*}\left(\left(z \epsilon_{k}\right)^{-1}\right)\right\} .
\end{aligned}
$$

Using $W(z)=W\left(z \epsilon_{k}\right)$ this results in

$$
W(z)=\frac{\sum_{k=0}^{D-1} \frac{1}{D} \frac{z^{D}-1}{z \epsilon_{k}-1}\left\{T^{*}(1)-T^{*}\left(\left(z \epsilon_{k}\right)^{-1}\right)\right\}}{1-\sum_{k=0}^{D-1} \frac{1}{D} \frac{z^{D}-1}{z \epsilon_{k}-1} B\left(z \epsilon_{k}\right) T\left(\left(z \epsilon_{k}\right)^{-1}\right)} .
$$

This does not represent a complete solution for $W(z)$ yet, as we do not have an exact expression for $T^{*}(z)$. However, making use of Rouché's theorem, we can overcome this last obstacle.

\subsection{Applying Rouché's theorem}

In order to apply Rouché's theorem, we need to reformulate the denominator of $W(z)$. More precisely, we rewrite the denominator as the sum of two analytic functions of $z$ in the domain $|z|<1$. Since the factor $T\left(\left(z \epsilon_{k}\right)^{-1}\right)$ has poles in this domain, we make further assumptions on the form of $T(z)=N(z) / P(z)$ to remove these poles. As for the degree of $N(z)$ and $P(z)$, two possibilities occur: either $N \leq P$, or $N>P$. Derivations for both cases follow a similar line, we confine ourselves here to the case $N \leq P$. Further, we write $P(z)$, the common denominator of $T(z)$ and $T^{*}(z)$, as

$P(z)=\prod_{j=1}^{C}\left(z-\gamma_{j}\right)^{m_{j}}$

(This notation is also adopted in Appendix.) The $\gamma_{j}(j=$ $1 \ldots C$ ) are the $C$ different zeroes of $P(z)$, all outside of the domain $|z|<1$, each with associated multiplicity $m_{j}$ $(j=1 \ldots C)$, and thus $P=\sum_{j=1}^{C} m_{j}$. Now, we are in the position to remove the poles of $T\left(\left(z \epsilon_{k}\right)^{-1}\right)$, by multiplying both numerator and denominator of $W(z)$ in (13) with

$\prod_{k=0}^{D-1}\left(z \epsilon_{k}\right)^{P} P\left(\left(z \epsilon_{k}\right)^{-1}\right)$

which gives us, considering (6) and (8), for $W(z)$

$W(z)=\frac{\sum_{k=0}^{D-1} \frac{1}{D} \frac{z^{D}-1}{z \epsilon_{k}-1}\left\{\prod_{k=0}^{D-1}\left(z \epsilon_{k}\right)^{P} P\left(\left(z \epsilon_{k}\right)^{-1}\right) T^{*}(1)-\prod_{k=0}^{D-1}\left(z \epsilon_{k}\right)^{P} N^{*}\left(\left(z \epsilon_{k}\right)^{-1}\right)\right\}}{\prod_{k=0}^{D-1}\left(z \epsilon_{k}\right)^{P} P\left(\left(z \epsilon_{k}\right)^{-1}\right)-\sum_{k=0}^{D-1} \frac{1}{D} \frac{z^{D}-1}{z \epsilon_{k}-1} B\left(z \epsilon_{k}\right) \prod_{k=0}^{D-1}\left(z \epsilon_{k}\right)^{P} N\left(\left(z \epsilon_{k}\right)^{-1}\right)}$. 
This can be somewhat simplified (not shown here, but applied below), by remarking that

$$
\begin{aligned}
\prod_{k=0}^{D-1}\left(z \epsilon_{k}\right)^{P} P\left(\left(z \epsilon_{k}\right)^{-1}\right) & =\prod_{k=0}^{D-1} \prod_{j=1}^{C}\left(1-z \epsilon_{k} \gamma_{j}\right)^{m_{j}} \\
& =\prod_{j=1}^{C}\left(1-z^{D} \gamma_{j}^{D}\right)^{m_{j}}
\end{aligned}
$$

which is an application of the identity $x^{D}-a^{D}=\prod_{k=0}^{D-1}(x-$ $\left.\epsilon_{k} a\right)$ at $x=1$ and $a=z \gamma_{j}$. A second step to take is to split the denominator of (14), denoted $R(z)$, into two functions $R_{1}(z)$ and $R_{2}(z)$, as follows,

$R(z)=R_{1}(z)+R_{2}(z)$,

$R_{1}(z)=\prod_{j=1}^{C}\left(1-z^{D} \gamma_{j}^{D}\right)^{m_{j}}$

$R_{2}(z)=-\sum_{k=0}^{D-1} \frac{1}{D} \frac{z^{D}-1}{z \epsilon_{k}-1} B\left(z \epsilon_{k}\right) \prod_{k=0}^{D-1}\left(z \epsilon_{k}\right)^{P} N\left(\left(z \epsilon_{k}\right)^{-1}\right)$,

where we applied the simplification of (15) to obtain $R_{1}(z)$. Now, one can verify that $R_{1}(z)$ and $R_{2}(z)$ are both analytic in the domain $|z|<1$ : the expression $\left(z \epsilon_{k}-1\right)$ is not an actual pole due to $\left(z^{D}-1\right), B(z)$ is analytic in this domain, and $\prod_{k=0}^{D-1}\left(z \epsilon_{k}\right)^{P} N\left(\left(z \epsilon_{k}\right)^{-1}\right)$ is too, since $N \leq P$.

In a third and last step, we remark that

$R\left(z \epsilon_{k}\right)=R(z) ; \quad R_{1}\left(z \epsilon_{k}\right)=R_{1}(z) ;$

$R_{2}\left(z \epsilon_{k}\right)=R_{2}(z) \quad(k=0, \ldots, D-1)$.

(For $R_{1}(z)$, this is obvious; for $R_{2}(z)$, this can be understood if one compares with the form of $W(z)(10)$, which also has this property.) Denoting $y=z^{D}$, we introduce equivalent functions $\hat{R}(y), \hat{R}_{1}(y)$ and $\hat{R}_{2}(y)$ for which

$\hat{R}\left(z^{D}\right)=R(z) ; \quad \hat{R}_{1}\left(z^{D}\right)=R_{1}(z) ; \quad \hat{R}_{2}\left(z^{D}\right)=R_{2}(z)$

Now, we take up the approach of [15], and apply Rouché's theorem on $\hat{R}(y)$. To do this, it is necessary that $\hat{R}_{1}(y)$ and $\hat{R}_{2}(y)$ (i) are analytic functions in $|y|<1$, (ii) are continuous at the boundary $|y|=1$, (iii) have derivatives at $y=1$, (iv) comply with the relation $\left|\hat{R}_{1}(y)\right|>\left|\hat{R}_{2}(y)\right|$ at $(|y|=1, y \neq 1),(\mathrm{v})$ comply with the relation $\hat{R}_{1}(1)=$ $-\hat{R}_{2}(1) \neq 0$, (vi) comply with the relation

$\frac{\hat{R}_{1}^{\prime}(1)+\hat{R}_{2}^{\prime}(1)}{\hat{R}_{1}(1)}>0$

where primes denote derivates.

If we assume the involved pgf's to be properly defined, and require the system to be stable, it can be easily checked that these six conditions are fulfilled. (What happens for an unstable system is explained in Sect. 4.1.) Invoking the theorem in [15], we conclude that the number of zeroes $N_{\hat{R}}$ of $\hat{R}(y)$ in the domain $|y|<1$ relates to the number of zeroes $N_{\hat{R}_{1}}$ of $\hat{R}_{1}(y)$ in the same domain (which is known to be $P$ ) according to the relation

$N_{\hat{R}}=N_{\hat{R}_{1}}-1=P-1$.

Including now the zero at $y=1$, the total number of zeroes for $\hat{R}(y)$ in the domain $|y| \leq 1$ sums up to $P$. Invoking that $W\left(z^{D}\right)$ is a proper pgf, with no singularities in the mentioned domain, all $P$ zeroes $y=\beta_{i}(i=0, \ldots, P-1)$ of the denominator $\hat{R}(y)$ in the domain $|y| \leq 1$ have to be compensated by the same zeroes $y=\beta_{i}$ in the numerator. Aware of these zeroes in the numerator, and using the knowledge that the numerator of $W\left(z^{D}\right)$ is of degree $P$ (which can be understood if one combines (14) and (15)), we cast $W\left(z^{D}\right)$ in a form

$W(z)=\frac{K^{*}\left(z^{D}-1\right)}{\hat{R}\left(z^{D}\right)} \prod_{i=1}^{P-1} \frac{z^{D}-\beta_{i}}{1-\beta_{i}}$.

Again, this expression reflects that $W(z)$ is a function of $z^{D}$. The constant $\beta_{0}$ is the zero of $\hat{R}(y)$ equal to one, while the other $\beta_{i}, i=1, \ldots, P-1$ are zeroes of $\hat{R}(y)$ in the domain $|y| \leq 1$. Since $\hat{R}(y)$ has poles $\beta_{i}, R(z)$ has $D \cdot P$ poles $\alpha_{j}$, $j=0, \ldots, D P-1$, that all fulfill the condition $\alpha_{j}^{D}=\beta_{i}$.

Note that, at this point, we indeed ruled out $T^{*}(z)$. The constant $K^{*}$ can be determined by demanding that $W(1)=1$ (normalization condition), and results in

$K^{*}=\prod_{j=1}^{C}\left(1-\gamma_{j}^{D}\right)^{m_{j}} \cdot\left\{\frac{\mathrm{E}[T]-B_{\mathrm{eq}}}{D}\right\}$

with $B_{\text {eq }}$ the equivalent burst length, defined as

$B_{\mathrm{eq}}=\mathrm{E}[B]+\frac{D-1}{2}+\sum_{k=1}^{D-1} \frac{B\left(\epsilon_{k}\right) T\left(\epsilon_{k}^{-1}\right)}{\epsilon_{k}-1}$.

Now, the pgf of the waiting time $W(z)$ is fully known. It then suffices to substitute $W(z)$ in (12), to obtain an explicit formula for $H(z)$, which was our aim.

\section{Derived measures}

In this section, we first define an equivalent load, that serves to characterize the infinite system's stability. Next, we take a look at approximate measures that allow to evaluate the loss performance of a finite-sized buffer. These we apply in Sect. 5. 


\subsection{Equivalent load}

As for the condition to stability, it can be intuitively understood that, for a buffer with infinite size, the queue length grows unbounded if the load is too high. For degenerate buffers, this happens even before the classic load $\rho=\mathrm{E}[B] / \mathrm{E}[T]$ reaches unity. We can characterize a case of unbounded growth by

$\lim _{n \rightarrow \infty} \operatorname{Pr}\left[H_{n}=0\right]=0$.

Considering (12) and (17), this occurs when $K^{*}$ (given by (18)) becomes zero, which happens when E[T] equals $B_{\text {eq }}$. From this, one can define a maximum tolerable arrival intensity $\lambda_{\max }$, that puts an upper limit to the arrival intensity $\lambda$, defined as $1 / \mathrm{E}[T]$. It follows that $\lambda_{\max }$ is the solution to the implicit expression (implicit, as $\lambda$ also occurs in the expression for $T(z))$

$\frac{1}{\lambda_{\max }}=\mathrm{E}[B]+\frac{D-1}{2}+\sum_{k=1}^{D-1} \frac{B\left(\epsilon_{k}\right) T\left(\epsilon_{k}^{-1}\right)}{\epsilon_{k}-1}$.

The symbols $\epsilon_{k}$ still represent the $D$ different complex $D$ th roots of unity, as in (10). The solution is thus function of the FDL granularity D, the (complete) pgfs of both interarrival and burst-size distribution, and can be found from (19) with a simple bisection algorithm. Related, we can define an equivalent load, that is given by

$\rho_{\mathrm{eq}}=\lambda \cdot\left(\mathrm{E}[B]+\frac{D-1}{2}+\sum_{k=1}^{D-1} \frac{B\left(\epsilon_{k}\right) T\left(\epsilon_{k}^{-1}\right)}{\epsilon_{k}-1}\right)$.

Now, the effect of voids is incorporated into an altered definition of the load. Remark that $\rho_{\text {eq }}$ equals one when the arrival intensity reaches $\lambda_{\max }$, which in general happens for a classic load smaller than one. Degenerate buffers are thus unstable for lower loads, if compared to classical (nondegenerate) buffers.

A final note goes to an alternative method to obtain $\lambda_{\max }$. One easily verifies that the conditions to apply Rouché's theorem in Sect. 3.5 are violated when $\lambda=\lambda_{\max }$. More precisely, the assumption that the system is stable then no longer holds, and the numerator of (16) turns zero, reflecting the system's unstability.

\subsection{Heuristic}

Results up to now are valid for a degenerate buffer of infinite size. To derive the loss probability for a buffer of finite size $N+1$, we rely on a heuristic called "heuristic B" in [9], that is defined as

$B L P \approx\left(1-\rho_{\mathrm{eq}}\right) \cdot \frac{\operatorname{Pr}\left[H_{\infty}>N \cdot D\right]}{1-\operatorname{Pr}\left[H_{\infty}>N \cdot D\right]}$.
Here, $H_{\infty}$ denotes the scheduling horizon in an infinite degenerate buffer (before denoted simply $H$ ) and $\rho_{\text {eq }}$ is the above-mentioned equivalent load. The overflow probabilities $\operatorname{Pr}\left[H_{\infty}>N \cdot D\right]$ can be obtained in many different ways from the expression of $H(z)$; here, we choose to apply a dominant pole approximation. To do this, we have to impose some additional (rather general) restrictions on the distribution of $B(z)$. A sufficient condition, e.g., is that $B$ has a rational pgf. We will adopt this convention from here on, and note that it poses no problem for the application of Sect. 5 . (Remark, however, that the model up to now is valid for any $B(z)$ with $\mathrm{E}[B]<\infty$.)

Under this assumption, tail probabilities have a quasigeometrical tail decay, with decay rate $z_{0}$, as

$\operatorname{Pr}\left[H_{\infty}>N \cdot D\right] \approx \frac{c s t}{z_{0}^{N \cdot D+1}}$,

with $z_{0}$ the (single) dominant pole of $H(z)$ along the positive real axis. The constant follows from the application of residue theory and is, in its final form, given by

$c s t=-\frac{1}{z_{0}} \lim _{z \rightarrow z_{0}}\left(W(z) \cdot\left(z-z_{0}\right)\right) \frac{D}{z_{0}^{D}-1}$.

The limit in the above can easily be calculated explicitly. As for the pole $z_{0}$, the function $W(z)$ relates to $H(z)$ according to (12) and both have the same dominant poles. Since for the latter, we had that $W(z)=W\left(z \epsilon_{k}\right)$, it is easy to see that there will be $D$ dominant poles, of the form $z_{k}=z_{0} \epsilon_{k}$ $(k=0, \ldots, D-1)$, with $z_{0}$ being (by definition) the positive real one. That the latter does indeed exist (under the assumptions discussed in Sect. 2.4), follows readily by inspecting the denominator of $W(z)$ (see (14)) along the positive real axis.

Although all formulas were found under the assumption of a stable system, note that the heuristic also performs well for overloaded systems, that is, with $\rho_{\mathrm{eq}}>1$. For a motivation hereof, see [11] (Sect. 3.4).

\section{Numerical comparison}

In this section, we perform a numerical comparison of simulation results against results from the heuristic of Sect. 4. Our scope is twofold. On the one hand, we want to assess the accuracy of the heuristic. On the other hand, we are particularly interested in the impact of correlation in the arrival process on the performance of an optical buffer, since arrival processes in (optical) communication networks are bursty, as noted before.

The buffer we consider has a fixed size $N=20$ and feeds into a single channel. Arriving bursts have a fixed service time $B$ of 20 time slots. The load $\rho=\mathrm{E}[B] / \mathrm{E}[T]$ remains 
Fig. 2 Simulation traces of the 5 runs, ordered (1 to 5) from top to bottom

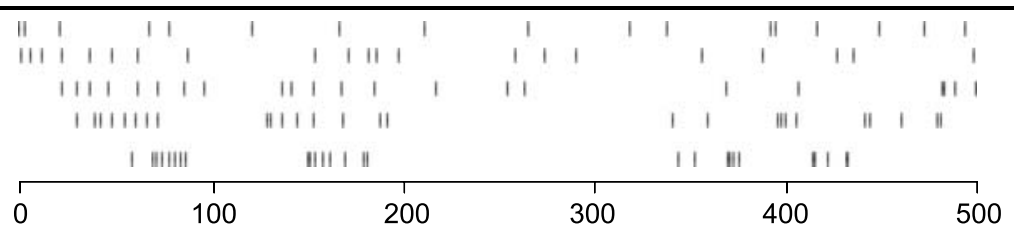

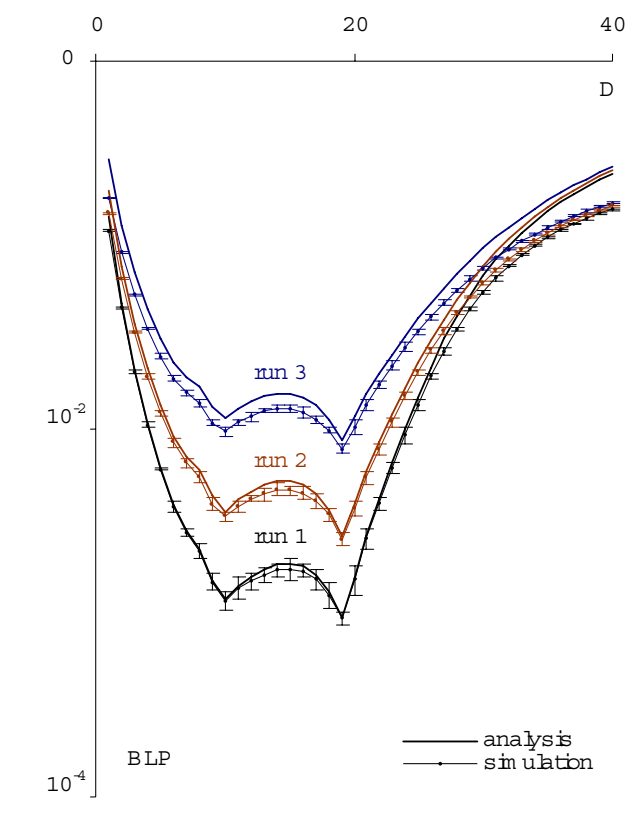

0

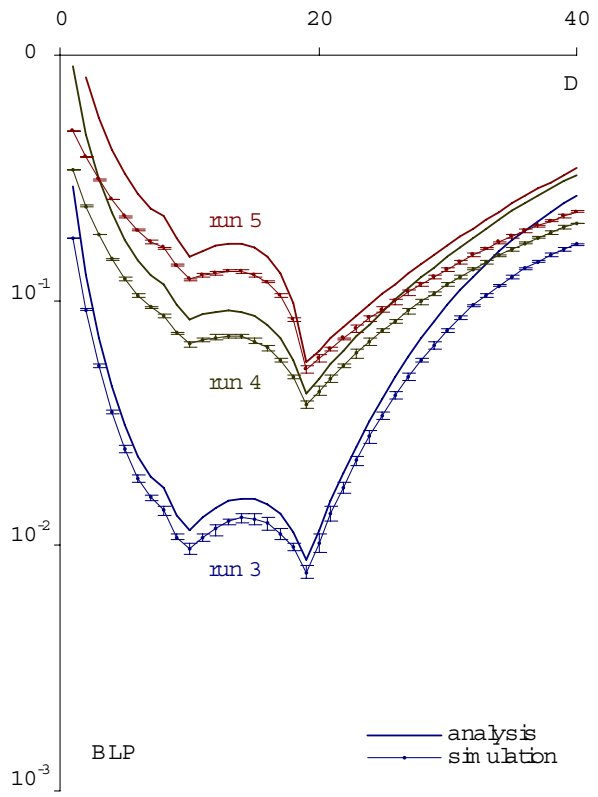

Fig. 3 Loss probability vs. granularity, for runs 1 to 5

(1)

.

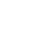

fixed to 60 percent, and thus also $E[T]$ is fixed. Now, we consider a correlation model for the inter-arrival times that uses a probabilistic mix of geometrically distributed rv's $T_{S}$ (parameter $p_{1}$ ) and $T_{L}$ (parameter $p_{2}$ ). More precisely, the pgf of the inter-arrival time distribution is given by

$T(z)=\alpha T_{S}(z)+(1-\alpha) T_{L}(z)$,

where $\alpha, 0 \leq \alpha \leq 1$, is a weighing factor, and $T_{S}(z)$ and $T_{L}(z)$ are the pgf's of $T_{S}$ and $T_{L}$ respectively. This model is able to capture an arrival process that we describe with "trains" and "wagons". That is, customers or "wagons" do not arrive independently (as in the case of a Bernoulli arrival process or a Poisson arrival process), but rather, they arrive in smaller or larger groups or "trains". The time inbetween customers of the same group, or "wagon spacing", generally denoted by $T_{S}$, is on average much smaller than the time between two trains or "train spacing", generally denoted by $T_{L}$. The average group size is captured by $G_{\text {avg }}=1 /(1-\alpha)$, expressing the average number of customers per group or "wagons per train". The average wagon spacing is captured by $S_{\text {avg }}=1 / p_{1}$, the average train spacing by $1 / p_{2}$ (both expressed in slots).

In Fig. 2, a sample trace of five different simulation runs is displayed, each with sample length 500 time slots. With each new run (increasing run number) parameter values were altered, so as to go from no correlation between
Table 1 Parameter setting used for the five runs

\begin{tabular}{lrrrrr}
\hline Run & 1 & 2 & 3 & 4 & 5 \\
\hline$G_{\text {avg }}$ & 1 & 2 & 4 & 4 & 4 \\
$S_{\text {avg }}$ & 20 & 20 & 20 & 10 & 5 \\
\hline
\end{tabular}

arrivals (run 1) to an increasing amount of correlation (run 2 to 5). The parameter setting for each run is displayed in Table 1. As can also be understood from the figure, first we augmented the average group size $G_{\text {avg }}$ (run 1 to 3), then we diminished the wagon spacing $S_{\text {avg }}$ (run 3 to 5). Note that, for all five runs, the load, the overall average inter-arrival time and the service time remained fixed.

Figure 3 displays the loss probability for varying granularity $D$ (in time slots), for each of the five runs. The results from the analysis are displayed as continuous curves, the simulations as discrete points connected with dotted lines, with a 95 percent confidence interval indicated for each simulation point.

As for the performance of the heuristic, it is clear that it attains high accuracy. Therefore, the analytic curve for run 1 matches the simulations very well, while for run 5 , the match is less striking. Nevertheless, it is clear that the discrepancy between simulation and analysis is very small around the optimum, where the loss probability is minimal.

As for the impact of correlation in the arrival process on the loss performance of a finite-sized degenerate buffer, 
the observation of increased loss comes rather as expected. Less evident is what happens to the optima. For fixed service times, previous work showed that the well-pronounced optima around $D_{1}=B-1$ and $D_{2}=(B-1) / 2$ remain optimal for a broad range of the load $\rho$ if arrivals occur independently. More precisely, it is known that $D_{1}$ is optimal for low values of the load (e.g. 10 percent) and the loss for $D_{2}$ is then much higher, while for a transition load $\rho \geq 0.652$ (for the assumed burst size), $D_{2}$ becomes optimal. Interestingly, the left pane of Fig. 3 (together with results not shown here) learns that this balance between $D_{1}$ and $D_{2}$ is hardly influenced when we increase the average group size in run 1 to 3: although the overall loss increases, the relative position of $D_{1}$ and $D_{2}$ is hardly influenced. Entirely different is the situation for run 3 to 5, as inspection of the right pane of Fig. 3 illustrates. Apparently, decreasing the wagon spacing has a devastating effect on the optimum at $D_{2}$, while the optimum $D_{1}$ is much less influenced. As such, correlated arrivals with small wagon spacing have the optimum $D_{1}$ for both low and higher loads.

Although not self-evident, this can be intuitively understood when we think of a specific situation with $D=D_{1}=$ $B-1=19$, with $B$ the fixed service time of 20 time slots. If an arriving burst is queued in delay line $j . D$, and the next burst arrives just 1 slot later, then this next burst is sent to delay line $(j+1) . D$ and there will be no time in-between the transmission of both bursts, implying that no void occurs. This situation is indeed likely in run 4 and $5(\operatorname{Pr}[T=1]$ is 0.077 and 0.152 respectively), while it is rather rare in run $1-3(\operatorname{Pr}[T=1]$ is $0.030,0.036$ and 0.041 respectively). If the arrival would occur not 1 , but 2 slots later, then the void size is still just 1 slot. Generalizing, the granularity value $D_{1}$ leads to small voids, especially when the case of a very small inter-arrival time is a likely one. And, since minimizing the void size results in better loss performance, one can see how $D_{1}$ outperforms $D_{2}$ on the right pane of Fig. 3, as $\operatorname{Pr}[T=1]$ increases. As a result, the optimum $D_{1}$ is more stable than it was under uncorrelated arrivals, and remains optimal also for higher loads. This reveals how special features of the inter-arrival time distribution, such as $\operatorname{Pr}[T=1]$, play an important role in the performance evaluation of degenerate buffers.

\section{Conclusions}

We presented an analytic model for a degenerate buffer of infinite size, with general inter-arrival and service times. We started from a single system equation, and then divided the problem into two parts. After treating the queueing effect and granularity effect separately, we combined solutions, and applied Rouché's theorem to determine the remaining unknowns. This yielded closed-form expressions for the pgf's of the scheduling horizon and the waiting time.
Further, we were able to characterize the system's stability with a maximum tolerable arrival intensity, and an equivalent load. Also, we proposed heuristics to quantify the loss performance of a finite-sized degenerate buffer, and confronted them with simulation results. The match was good, especially around the optima. Also, the results allowed for a deeper insight on the impact of correlation in the arrival process on loss performance, and the importance of special features of the inter-arrival time distribution in that context.

In future work, our main challenge is the extension that would allow for multiple channels serving a shared degenerate buffer. Evidently, that analysis encounters the same mathematical problems as the ones known from conventional multi-server queues. Therefore, we aim to come up with approximations, that do justice to the specific nature of degenerate (or optical) buffers.

\section{Appendix}

In this appendix, we explain how to solve the queueing effect (5)

$H=[G-T]^{+}$,

in terms of pgf's, in a way similar to the approach in [14]. As mentioned above, the solution will contain an auxiliary function $T^{*}(z)$. In the first step, we rewrite the $\operatorname{pgf}$ of $T$, so as to facilitate the analysis of the second step.

Rewriting the pgf of $T$

The pgf of $T(z)$ mentioned in (6) can be rewritten as follows.

$$
\begin{aligned}
T(z) & =\sum_{i=0}^{R} r_{i} z^{i}+\frac{\sum_{i=0}^{Q} q_{i} z^{i}}{\prod_{j=1}^{C}\left(z-\gamma_{j}\right)^{m_{j}}} \\
& =\sum_{i=0}^{R} r_{i} z^{i}+\sum_{j=1}^{C} \sum_{i=0}^{m_{j}-1} \frac{b_{i j}}{\left(\gamma_{j}-z\right)^{i+1}} .
\end{aligned}
$$

Subsequently we isolate the polynomial part of the fraction, to perform then a partial fraction expansion. The $\gamma_{j}$ $(j=1, \ldots, C)$ are the $C$ different poles of $T(z)$, each with associated multiplicity $m_{j}(j=1, \ldots, C)$. Putting to use the probability generating property of a pgf, one can express the probabilities $t_{k}(k=0,1 \ldots)$, corresponding to $T(z)$, as

$$
\begin{aligned}
t_{k} & =\left.\frac{1}{k !} \frac{\partial^{k}}{\partial z^{k}} T(z)\right|_{z=0} \\
& =\sum_{i=0}^{R} r_{i} \delta\langle i-k\rangle+\sum_{j=1}^{C} \sum_{i=0}^{m_{j}-1}\left(\begin{array}{c}
i+k \\
i
\end{array}\right) \frac{b_{i j}}{\gamma_{j}^{i+k+1}},
\end{aligned}
$$


where $\delta\langle i-k\rangle$ denotes the discrete delta function, that equals 1 if $i=k$, and zero if $i \neq k$. Reordering the binomial coefficients $\left(\begin{array}{c}i+k \\ i\end{array}\right)$, and introducing coefficients $c_{i j}$, we arrive at

$t_{k}=\sum_{i=0}^{R} r_{i} \delta\langle i-k\rangle+\sum_{j=1}^{C} \sum_{i=0}^{m_{j}-1} \frac{k^{i}}{\gamma_{j}^{k}} c_{i j}$.

Obtaining the pgf of $H$

With the definition of a pgf as a starting point, we apply (20) to obtain

$$
\begin{aligned}
H(z) & =\sum_{m=0}^{\infty} h_{m} z^{m} \\
& =\sum_{n=0}^{\infty} \sum_{k=0}^{\infty} g_{n} t_{k} z^{[n-k]^{+}} \\
& =\sum_{n=0}^{\infty} \sum_{k=0}^{\infty} g_{n} t_{k} z^{n-k}+\sum_{n=0}^{\infty} \sum_{k=n}^{\infty} g_{n} t_{k}\left(z^{0}-z^{n-k}\right) \\
& =G(z) T\left(z^{-1}\right)+\sum_{k=0}^{\infty}\left(1-z^{-k}\right) \sum_{n=0}^{\infty} g_{n} t_{k+n},
\end{aligned}
$$

where $g_{n}$ and $t_{k}$ are the probabilities corresponding to $G(z)$ and $T(z)$ respectively, and $[x]^{+}$is shorthand for $\max \{x, 0\}$. We introduce the coefficients $t_{k}^{*}(k=0, \ldots, \infty)$,

$$
\begin{aligned}
t_{k}^{*} & =\sum_{n=0}^{\infty} g_{n} t_{k+n} \\
& =\sum_{n=0}^{\infty} g_{n}\left(\sum_{i=0}^{R} r_{i} \delta\langle k+n-i\rangle+\sum_{j=1}^{C} \sum_{m=0}^{m_{j}-1} \frac{(k+n)^{m}}{\gamma_{j}^{k+n}} c_{m j}\right),
\end{aligned}
$$

where we used (22) in the last transition. The first term in this expression we can rewrite as

$$
\begin{aligned}
& \sum_{n=0}^{\infty} g_{n} \sum_{i=0}^{R} r_{i} \delta\langle k+n-i\rangle \\
& \quad=\sum_{i=0}^{\min (k, R)} r_{i} g_{i-k}=r_{k}^{*}=\sum_{i=0}^{R} r_{i}^{*} \delta\langle k-i\rangle,
\end{aligned}
$$

where we introduced the coefficients $r_{k}^{*}$ for notational convenience. The second term of (24) can be reformulated as

$$
\sum_{n=0}^{\infty} g_{n} \sum_{j=1}^{C} \sum_{m=0}^{m_{j}-1} \frac{(k+n)^{m}}{\gamma_{j}^{k+n}} c_{m j}
$$

$$
\begin{aligned}
& =\sum_{j=1}^{C} \sum_{m=0}^{m_{j}-1} \sum_{n=0}^{\infty} g_{n} \sum_{i=0}^{m} \frac{\left(\begin{array}{c}
m \\
i
\end{array}\right) k^{i} n^{m-i}}{\gamma_{j}^{k+n}} c_{m j} \\
& =\sum_{j=1}^{C} \sum_{i=0}^{m_{j}-1} \frac{k^{i}}{\gamma_{j}^{k}}\left(\sum_{n=0}^{\infty} g_{n} \sum_{m=i}^{m_{j}-1} \frac{\left(\begin{array}{c}
m \\
i
\end{array}\right) n^{m-i}}{\gamma_{j}^{n}} c_{m j}\right) \\
& =\sum_{j=1}^{C} \sum_{i=0}^{m_{j}-1} \frac{k^{i}}{\gamma_{j}^{k}} c_{i j}^{*} .
\end{aligned}
$$

In the second step, we interchanged the sums over $m$ and $i$, respectively, while in the third step, we introduced other coefficients $c_{i j}^{*}$ for notational convenience.

Now, the form of the $t_{k}^{*}$ is captured by

$t_{k}^{*}=\sum_{i=0}^{R} r_{i}^{*} \delta\langle k-i\rangle+\sum_{j=1}^{C} \sum_{m=0}^{m_{j}-1} \frac{k^{m}}{\gamma_{j}^{k}} c_{m j}^{*}$,

which obviously resembles the form of the $t_{k}$ (22). If we now introduce the auxiliary function $T^{*}(z)$,

$T^{*}(z)=\sum_{k=0}^{\infty} t_{k}^{*} z^{k}$

the resemblance between (22) and (25) leads to the conclusion that $T(z)$ and $T^{*}(z)$ have a common denominator $P(z)$. Also, we see that the numerator of $T^{*}(z)$ has degree $N$ or less. The result of the queueing effect then follows from (23), leading to

$H(z)=G(z) T\left(z^{-1}\right)+T^{*}(1)-T^{*}\left(z^{-1}\right)$

which is the result we aimed for in this Appendix.

\section{References}

1. Lakatos, L.: On a simple continuous cyclic-waiting problem. Ann. Univ. Sci. Budapest. Sect. Comp. 14, 105-113 (1994)

2. Lakatos, L.: On a simple discrete cyclic-waiting queueing problem. J. Math. Sci. New York 4(92), 4031-4034 (1998)

3. Mykhalevych, K.V.: A comparison of a classical retrial M/G/1 queueing system and a Lakatos-Type M/G/1 cyclic-waiting time queueing system. Ann. Univ. Sci. Budapest. Sect. Comp. 23 (2003)

4. Artalejo, J.R.: A classical bibliography of research on retrial queues: progress in 1990-1999. TOP 7, 187-211 (1999)

5. Atencia, I., Moreno, P.: A discrete-time Geo/G/1 retrial queue with general retrial times. Queueing Syst. 48(1-2), 5-21 (2004)

6. Qiao, C., Yoo, M.: Optical Burst Switching-a new paradigm for an optical Internet. J. High-Speed Netw. 8, 69-84 (1999)

7. Callegati, F.: Optical buffers for variable length packets. IEEE Commun. Lett. 4(9), 292-294 (2000)

8. Murata, M., Kitayama, K.: Ultrafast photonic label switch for asynchronous packets of variable length. In: Proceedings of INFOCOM 2002, New York, June 2002

9. Laevens, K., Bruneel, H.: Analysis of a single-wavelength optical buffer. In: Proceedings of Infocom 2003, San Francisco 
10. Rogiest, W., Laevens, K., Fiems, D., Bruneel, H.: A performance model for an asynchronous optical buffer. Perform. Eval. 62(1-4), 313-330 (2005)

11. Laevens, K., Moeneclaey, M., Bruneel, H.: Queueing analysis of a single-wavelength Fiber-Delay-Line buffer. Telecommun. Syst. 31(2-3), 259-287 (2006)

12. Almeida, R.C., Pelegrini, J.U., Waldman, H.: Optical buffer modelling for performance evaluation considering any packet interarrival time distribution. In: IEEE International Conference on Communications, vol. 3, pp. 1771-1775, 2004
13. Almeida, R.C., Pelegrini, J.U., Waldman, H.: A generic-traffic optical buffer modeling for asynchronous optical switching networks. IEEE Commun. Lett. 9(2), 175-177 (2005)

14. Bruneel, H., Kim, B.G.: Discrete-Time Models for Communication Systems Including ATM. Kluwer Academic, Boston (1993)

15. Klimenok, V.I.: On the modification of Rouche's theorem for the queueing theory problems. Queueing Syst. 38(4), 431-434 (2001) 